\title{
Anesthetic Management of a Pregnant Female with Interstitial Lung Disease and Pulmonary Hypertension for Emergency Lower Segment Cesarean Section
}

\author{
Bhavna Gupta $^{1 *}$, Munisha Agarwal ${ }^{2}$ \\ ${ }^{1,2}$ Department of Anesthesia, MAMC and Lok Nayak Hospital, India
}

*Corresponding Author: Bhavna Gupta, Department of Anesthesia, MAMC and Lok Nayak Hospital, India, Email: bhavna.kakkar@gmail.com

\begin{abstract}
Preoperative risk assessment, optimization, successful anesthesia management of a pregnant female with interstitial lung disease with pulmonary hypertension is a meticulous task and it becomes more challenging in cases of emergency scenario where time is an essence. We describe the successful management of our case with the use of combined spinal epidural anesthesia. Our case was unique as it is rare to find an association of interstitial lung disease with severe pulmonary hypertension during pregnancy.
\end{abstract}

Keywords: Emergency lower segment cesarean section, pulmonary hypertension, interstitial lung disease, pregnant female, combined spinal epidural anesthesia

\section{INTRODUCTION}

Pulmonary arterial hypertension is defined as persistent increase in mean pulmonary artery pressure more than or equal to $25 \mathrm{mmHg}$ and pulmonary occlusion pressure less than 15 mmHg. Symptoms of patients include cough, breathlessness, syncope, weakness, fatigue, chest pain etc. Pulmonary hypertension of different causes leads to a common pathway of right ventricular strain pattern ultimately leading to right heart failure. All measures are done to avoid further rise in pulmonary arterial pressure and requires collaborative efforts of obstetrician, anesthesiologist, cardiologist, intensivists and pulmonologist. Preoperative evaluation and perioperative management takes in account the functional status of the patient, type of surgery involved, severity of disease and revolves around balancing the systemic vascular resistance and pulmonary vascular resistance.

\section{Case Presentation}

Thirty year old 36 weeks (gestational age) female, gravida 2 para 2 live 1 lady, a chronic chulha worker (since five years), was following up in obstetric out-patient department for her regular antenatal checkups. She had history of breathlessness which had progressed to dyspnea on rest, easy fatigue ability, chronic off and on cough with or without expectoration. On further evaluation of the general condition, she was diagnosed to have interstitial lung disease and bronchiectasis leading to severe pulmonary hypertension and was under regular follow-up since two years. She was admitted in hospital few times, owing to severe breathlessness and twice had history of right heart failure. She was taking inhaled and oral bronchodialtors, lasix, oxygen, and was managed conservatively. She was planned to undergo induction of labour at 37 weeks but presented to us for emergency lower segment cesarean section (LSCS) in view of transverse lie of fetus and fetal distress. She had last delivery 3 years back with uneventful normal delivery. Her arterial room air saturation was $86-88 \%$ and on $99 \%$ with oxygen. On auscultation, air entry was equal on both sides but there were coarse creptitations in bases of both lungs. Chest $\mathrm{X}$ ray showed prominent bronchovascular markings with prominent bat wing appearance and cardiomegaly suggestive of pulmonary edema. Echocardiography revealed severe tricuspid regurgitation, severe pulmonary hypertension, dilated right atrium and right ventricle. Patient was prepared to undergo lower segment caesarean section under combined spinal epidural (CSE) anesthesia and high risk consent was taken for the same with back up bed ventilator in ICU was arranged in case the need arise. She was given her usual bronchodilators in the preoperative area and was 
wheeled inside Operation Theatre on trolley with oxygen@6L/min. After attaching routine standard monitors according to ASA recommendations, she was made to sit comfortably for Combined Spinal Epidural anesthesia. Invasive Blood pressure monitoring could not be achieved owing to non-availability of arterial module. Epidural catheter was placed at L1-2 catheter fixed at $9 \mathrm{~cm}$, test dose was omitted initially, to titrate with the effect of subarachnoid blockade. Subarachnoid needle $27 \mathrm{G}$ was placed at L3-4 and total drug given was 1.5 cc $0.5 \%$ Heavy bupivacaine with 15 microgram fentanyl citrate. Level of achieved blockade was T8, and supplemented with test dose, $1.5 \%$ 3cc xylocaine, to achieve T5 level. The patient was positioned supine with left uterine displacement. A viable baby was delivered, and oxytocin was given as infusion at the rate of 5-10units/hour titrated to uterus contraction. Oxygen saturation was maintained between $98-99 \%$ by venti mask. After surgery, the patient was transferred to the high dependency unit. We gave bilateral transversus abdominis plane (TAP) block and morphine 1.5 mg via epidural catheter to provide adequate pain relief. Patient maintained stable vitals in HDU and was transferred to medicine ward for further follow up.

\section{DISCUSSION}

Pregnant females with interstitial lung disease, bronchiectasis and severe pulmonary hypertension for emergency LSCS is a challenge for the anesthesiologists. Thorough understanding of pathophysiology, severity of pulmonary hypertension, treatment modality is required to deliver optimal care to a pregnant female for emergency LSCS. Parturient females are at increased risk of aspiration because of incompetent lower esophageal sphincter and distortion of gastric anatomy. Pregnant females also have a difficult airway both for mask ventilation and intubation owing to physiological changes. There is also capillary engorgement of respiratory mucosa making them more friable. There is also decrease in functional residual capacity and increase in oxygen consumption which result in the rapid development of hypoxemia during periods of hypoventilation in parturient and more so in a compromised patient with decreased cardiopulmonary reserves. Pulmonary hypertension is defined as pulmonary artery pressure more than $25 \mathrm{mmHg}$ at rest. Important benefit of LSCS is it cuts short second stage of labor and avoids pain induced increase in pulmonary artery pressure. NYHA physical status classification is important for assessment and helps predicts morbidity and mortality. Routine standard monitoring including electrocardiogram, non invasive blood pressure and peripheral oxygen saturation is mandatory. Invasive blood pressure monitoring is indicated in severe pulmonary hypertensive patients undergoing surgery with major fluid shifts. [1] We avoided general anesthesia in our patient to avoid undue risk of pulmonary aspiration, rapid changes of systemic and pulmonary vascular resistance with a rapid sequence intubation. Positive pressure ventilation may decrease venous return and increase pulmonary vascular resistance. [1]

Goal of anesthesia management include maintenance of preload, systemic vascular resistance, contractility to maintain cardiac output and to prevent rise in pulmonary vascular resistance (hypoxia, hypercarbia, hypothermia, academia, pain).Systemic hypotension may also increase the degree of shunting and worsen hypoxia.[1]

We chose for CSE in our patient so as to avoid the unwanted effects of GA in our patient, and although central neuraxial blockade has been used safely in patients with $\mathrm{PH}$, blocking cardiac sympathetic fibers in the upper thoracic region disrupts right ventricular regulation and can be detrimental in parturient with pulmonary hypertension[1].Tight hemodynamic monitoring and control is of utmost importance. Fluid management is equally challenging as they poorly tolerate both hypo and hypervolemia. Epidural anesthesia has a slow onset about 15 minutes but allows for hemodynamic stability. Spinal anesthesia helps achieve adequate blockade quickly but at the expense of sudden hypotension, we therefore preferred low dose spinal with epidural anesthesia to achieve rapid blockade and maintaining hemodynamic stability. Vigilant monitoring by the anesthesiologist is necessary to ensure adequate treatment of hypotension.[2]

Postoperative pain control was also an important aspect of care to avoid increases in pulmonary hypertension. All attempts to reduce pulmonary hypertension prior to surgery should be performed, such as the administration of oxygen, bronchodilators, antibiotics, and steroids to the patient with obstructive lung disease. Post-operative complications or deterioration are common in post-operative 
period and they are at risk of fluid shifts, arrhythmia, pulmonary vasoconstriction, hypotension etc. majority of deaths occur in the first month of delivery, therefore clinical observation is mandatory. [1] Multimodal analgesia should be the norm in the immediate post-operative period and the same was provided via epidural analgesia and TAP blockade.

\section{PATIENT CONSENT Form}

We have obtained patient consent for the case report. The patients understand that their names and initials will not be published and due efforts have been made to conceal their identity.

\section{REFERENCES}

[1] Gupta B, Kakkar L, Gupta A. Anesthetic considerations for a parturient with pulmonary hypertension. Indian Anaesth Forum 2017; 18:39-45

[2] Meng ML, Landau R, Viktorsdottir O, Banayan J, Grant T, Bateman B, Smiley R, Reitman E. Pulmonary Hypertension in Pregnancy: A Report of 49 Cases at Four Tertiary North American Sites. Obstet Gynecol. 2017 Mar;129(3):511-520

Citation: Bhavna Gupta, Munisha Agarwal. Anesthetic Management of a Pregnant Female with Interstitial Lung Disease and Pulmonary Hypertension for Emergency Lower Segment Cesarean Section. ARC Journal of Anesthesiology. 2018; 3(1):18-20. DOI: dx.doi.org/10.20431/2455-9792.0301005.

Copyright: (C) 2018 Authors. This is an open-access article distributed under the terms of the Creative Commons Attribution License, which permits unrestricted use, distribution, and reproduction in any medium, provided the original author and source are credited. 